\title{
Efficient circular neighbour designs for spatial interference model
}

\author{
Pierre Druilhet* and Tinsson Walter** \\ *Laboratoire de Mathématiques, UMR CNRS 6620, Clermont Université, Université \\ Blaise Pascal, 63177 AUBIERE Cedex, France \\ **Laboratoire de Mathématiques Appliquées, UMR CNRS 5142, Université de Pau, BP \\ 1155, 64013 PAU Cedex, France
}

\begin{abstract}
We consider circular block designs for field-trials when there are two-sided spatial interference between neighbouring plots of the same blocks. The parameter of interest is total effects, that is the sum of direct effect of treatment and neighbour effects, which correspond to the use of a single treatment in the whole field. We determine universally optimal approximate designs. When the number of blocks may be large, we propose efficient exact designs generated by a single sequence of treatment. We also give efficiency factors of the usual binary block neighbour balanced designs which can be used when the number of blocks is small.
\end{abstract}

Keywords: Approximate design; Neighbour design; Optimal design; Total effect; Universal optimality.

\section{Introduction}

In agricultural experiments, the response on a given plot is often affected by the treatments applied to the neighbouring plots (see e.g. Besag and Kempton, 1986). For a variety of models with two sided neighbour effects, optimal or efficient neighbour designs are available for the estimation of direct or neighbour effects separately. Most often, when the number of plots per blocks is not greater than the number of treatment, these designs are binary blocks designs. For example, Druilhet (1999) shows that circular designs neighbour-balanced at distance 1 and 2 (CNBD2) are universally optimal for the estimation of direct treatment effects among all binary blocks designs and when the number of blocks is small, that some of them are universally optimal 
over all possible designs. Kunert (2001) and Filipiak and Markiewicz (2005) propose efficiency factors for CNDB2s. Kunert and Martin (2000) obtain optimal or efficient designs for designs without guard plots when the number of blocks is large.

In a field experiment, one of the aim is to select a single treatment or variety which will be used on larger spatial areas than those used for individual treatments in the experiment: for example, a single variety of wheat to be grown in whole fields. When the chosen treatment is in use, its only neighbour will be itself: thus the effect of most importance is the sum of the direct effect of the treatment and the neighbour effects of the same treatment, called total effects (see e.g. McGilchrist and Trenbath, 1971, Kempton, 1985 and 1997, Besag and Kempton, 1986). For such effect and for a model with one-sided neighbour effects or carry-over effects in the context of repeated measurement designs, Bailey and Druilhet (2004) obtain optimal designs generated by only one sequence by using an upper bound of the information matrix. Then, they deduce efficiency factors for non-optimal designs such as binary neighbour balanced designs that are used in practice. For a model with two-sided neighbour effects, they use the same kind of upper bound of the information matrix which is in fact too rough to obtain optimal designs or sharp efficiency factors. In this paper, we construct optimal designs in the context of approximate designs introduced by Kushner (1997) and adapted to total effects by Druilhet and Tinsson (2009). We also obtain the sharpest lower bound of efficiency factor for non-optimal designs. For example, we show that CNBD2 are efficient for the estimation of total effects especially when the number of plots per blocks is not too large. We also propose efficient non-binary block designs generated by a single optimal sequence which usually require a large number of blocks.

\section{The designs and the model}

We consider designs with $b$ blocks divided in $k$ plots per blocks. To avoid edge effects, a guard plot is added at each end of the blocks. Let $t$ be the number of treatments. For $1 \leq i \leq b$ and $1 \leq j \leq k$, denote by $d(i, j)$ the treatment assigned to block $i$ in plot $j$. We assume the parsimonious additive model for the response $y_{i j}$ :

$$
y_{i j}=\beta_{i}+\tau_{d(i, j)}+\lambda_{d(i, j-1)}+\rho_{d(i, j+1)}+\varepsilon_{i j},
$$


where $\beta_{i}$ is the effect of the block $i, \tau_{d(i, j)}$ is the effect of the treatment $d(i, j)$, $\lambda_{d(i, j-1)}$ and $\rho_{d(i, j+1)}$ are respectively the left and right neighbor effects, $\varepsilon_{i j}$ are independent identically distributed errors with expectation 0 and variance $\sigma^{2}$. In vector notation, the model can be written:

$$
Y=B \beta+T_{d} \tau+L_{d} \lambda+R_{d} \rho+\varepsilon
$$

where $Y$ is the vector of the $n=b k$ responses, $\varepsilon$ is the vector of the errors, $\beta, \tau, \lambda$ and $\rho$ correspond to, respectively, the vector of blocks effects, direct effects, left neighbor effects and right neighbor effects. The incidence matrices associated to these effects are respectively given by $B, T_{d}, L_{d}$ and $R_{d}$ when the design $d$ is used. Note that $B=I_{b} \otimes \mathbb{I}_{k}$, where $I_{b}$ is the identity matrix of order b, the symbol $\otimes$ denotes Kronecker product and $\mathbb{I}_{k}$ is the k-dimensional vector of ones. We assume that $\mathbb{E}(\varepsilon)=0$ and $\operatorname{Var}(\varepsilon)=\sigma^{2} I_{b k}$. We denote the vector $\phi$ of total effects by $\phi=\tau+\lambda+\rho$ which corresponds to the situation when only one selected treatment is used (its neighbors are then itself). Denote $\theta^{\prime}=\left(\tau^{\prime}, \lambda^{\prime}, \rho^{\prime}\right)$ and $K^{\prime}=\left(I_{t}\left|I_{t}\right| I_{t}\right)$, then:

$$
\phi=K^{\prime} \theta
$$

We consider in this paper only circular designs, i.e. designs such that the treatment applied to a guard plot is the same treatment which is applied at the opposite end of the block. So, we have $d(i, 0)=d(i, k)$ and $d(i, k+1)=$ $d(i, 1)$, where $d(i, 0)$ and $d(i, k+1)$ are the treatments applied to the left and right guard plots of block $i$. It is worth noting that all the techniques proposed in his paper to derive optimal approximate designs can be easily adapted to designs without guard plots or models with more general intrablock covariance structure of the errors.

\section{Information matrices}

\subsection{Information matrix for $\theta$}

Denote $\omega_{B}=B\left(B^{\prime} B\right)^{-1} B^{\prime}$ the projection matrix onto the column span of $B$ and $\omega_{B}^{\perp}=I_{n}-\omega_{B}$. The information matrix $C[\theta]$ of the vector $\theta$ is given by (see e.g. Kunert, 1983):

$$
C_{d}[\theta]=\left(T_{d}\left|L_{d}\right| R_{d}\right)^{\prime} \omega_{B}^{\perp}\left(T_{d}\left|L_{d}\right| R_{d}\right) .
$$


i.e.

$$
C_{d}[\theta]=\left(\begin{array}{ccc}
T_{d}^{\prime} \omega_{B}^{\perp} T_{d} & T_{d}^{\prime} \omega_{B}^{\perp} L_{d} & T_{d}^{\prime} \omega_{B}^{\perp} R_{d} \\
L_{d}^{\prime} \omega_{B}^{\perp} T_{d} & L_{d}^{\prime} \omega_{B}^{\perp} L_{d} & L_{d}^{\prime} \omega_{B}^{\perp} R_{d} \\
R_{d}^{\prime} \omega_{B}^{\perp} T_{d} & R_{d}^{\prime} \omega_{B}^{\perp} L_{d} & R_{d}^{\prime} \omega_{B}^{\perp} R_{d}
\end{array}\right)=\left(\begin{array}{ccc}
C_{d 11} & C_{d 12} & C_{d 13} \\
C_{d 12}^{\prime} & C_{d 22} & C_{d 23} \\
C_{d 13}^{\prime} & C_{d 23}^{\prime} & C_{d 33}
\end{array}\right)
$$

By circularity of the design, $T_{d}^{\prime} T_{d}=L_{d}^{\prime} L_{d}=R_{d}^{\prime} R_{d}$ and $B^{\prime} T_{d}=B^{\prime} L_{d}=B^{\prime} R_{d}$. Therefore:

$$
C_{d 11}=C_{d 22}=C_{d 33}
$$

Since $T_{d} \mathbb{I}_{t}=L_{d} \mathbb{I}_{t}=R_{d} \mathbb{I}_{t}=\mathbb{I}_{n}$ and $\mathbb{I}_{n} \in \operatorname{Range}(B)$, we have:

$$
\forall i, j=1,2,3, C_{d i j} \mathbb{I}_{t}=0 \text {. }
$$

Consider the $(k \times k)$ permutation matrix

$$
V=\left(\begin{array}{ccccc}
0 & 0 & 0 & \ldots & 1 \\
1 & 0 & \ddots & & 0 \\
0 & 1 & \ddots & \ddots & 0 \\
\vdots & \ddots & \ddots & 0 & 0 \\
0 & \ldots & 0 & 1 & 0
\end{array}\right),
$$

then $L_{d}=\left(I_{b} \otimes V\right) T_{d}$ and $R_{d}=\left(I_{b} \otimes V^{\prime}\right) T_{d}$ and $V J_{k}=J_{k}=J_{k} V$ with $J_{k}=\mathbb{I}_{k} \mathbb{I}_{k}^{\prime}$ (see e.g. Kunert, 2001). We also have $\omega_{B}^{\perp}=I_{b} \otimes Q_{k}$ with $Q_{k}=$ $\omega_{\mathbb{I}_{k}}^{\perp}=I_{k}-k^{-1} J_{k}$ and then:

$$
C_{d 12}^{\prime}=C_{d 13}
$$

We denote by $T_{d u}, L_{d u}$ and $R_{d u}$ the incidence matrices restricted to block $u$. Thus $T_{d}^{\prime}=\left(T_{d 1}^{\prime}|\ldots| T_{d b}^{\prime}\right), L_{d}^{\prime}=\left(L_{d 1}^{\prime}|\ldots| L_{d b}^{\prime}\right), R_{d}^{\prime}=\left(R_{d 1}^{\prime}|\ldots| R_{d b}^{\prime}\right)$ and the blocks of $C_{d}[\theta]$ are given by:

$$
\begin{aligned}
& C_{d 11}=C_{d 22}=C_{d 33}=T_{d}^{\prime}\left(I_{b} \otimes Q_{k}\right) T_{d}=\sum_{u=1}^{b} T_{d u}^{\prime} Q_{k} T_{d u} \\
& C_{d 12}=C_{d 13}^{\prime}=T_{d}^{\prime}\left(I_{b} \otimes Q_{k}\right) L_{d}=\sum_{u=1}^{b} T_{d u}^{\prime} Q_{k} L_{d u} \\
& C_{d 23}=L_{d}^{\prime}\left(I_{b} \otimes Q_{k}\right) R_{d}=\sum_{u=1}^{b} L_{d u}^{\prime} Q_{k} R_{d u} .
\end{aligned}
$$




\subsection{Information matrix for total effects $\phi$}

The information matrix for the parameter of interest $\phi$ may be obtained from $C[\theta]$ by the extremal representation (see Gaffke, 1987 or Pukelsheim, 1993):

$$
C_{d}\left[K^{\prime} \theta\right]=\min _{L \in \mathbb{R}^{3 t \times t} / L^{\prime} K=I_{t}} L^{\prime} C_{d}[\theta] L,
$$

where the minimum is taken relative to the Loewner ordering. The minimum exists and is unique for a given design $d$ and a given effect $K$. Denote by $L^{*}$ a $3 t \times t$ matrix that minimizes $L^{\prime} C_{d}[\theta] L$ under the constraint $L^{\prime} K=I_{t}$. Then:

$$
C_{d}\left[K^{\prime} \theta\right]=L^{* \prime} C_{d}[\theta] L^{*} .
$$

Pukelsheim (1993) proposed general methods to obtain $L^{*}$ but they are not easily tractable for our purpose. In the following, we will see that optimal designs are symmetric designs: a design $d$ is said to be symmetric if all the blocks $C_{d i j}$ are completely symmetric, i.e. $C_{d i j}=a_{i j} I_{t}+b_{i j} J_{t}$ for some scalars $a_{i j}$ and $b_{i j}$. In that case, Druilhet and Tinsson (2009, Corollary 1) show that $L^{*}$ has the simple form $L^{*^{\prime}}=\left(a_{1}^{*} I_{t}\left|a_{2}^{*} I_{t}\right| a_{3}^{*} I_{t} \mid\right)$ for some scalars $a_{1}^{*}, a_{2}^{*}$ and $a_{3}^{*}$. In order to satisfy the constraint $L^{\prime} K=I_{t}$ with $K=\mathbb{I}_{3} \otimes I_{t}$ the matrix $L^{*}$ is then given by

$$
L^{* \prime}=\left((1-x-y) I_{t}\left|x I_{t}\right| y I_{t}\right),
$$

for some scalars $x$ and $y$.

\section{Linearization of the problem}

Since there exists a unique global minimum in (1), $L^{*}$ minimizes $L^{\prime} C_{d}[\theta] L$ if and only if $L^{*}$ minimizes $\operatorname{tr}\left(L^{\prime} C_{d}[\theta] L\right)$, both minimizations under the constraint $L^{\prime} K=I_{t}$. Therefore, we denote:

$$
q_{d}(x, y)=\operatorname{tr}\left(L^{* \prime} C_{d}[\theta] L^{*}\right) .
$$

From (2), we obtain for every symmetric design:

$$
\begin{aligned}
L^{* \prime} C_{d}[\theta] L^{*}= & 2\left(C_{d 11}-C_{d 12}\right) x^{2}+2\left(C_{d 11}-C_{d 12}\right) y^{2}+ \\
& 2\left(C_{d 11}-2 C_{d 12}+C_{d 23}\right) x y- \\
& 2\left(C_{d 11}-C_{d 12}\right) x-2\left(C_{d 11}-C_{d 12}\right) y+C_{d 11} .
\end{aligned}
$$


Denoting $c_{d i j}=\operatorname{tr}\left(C_{d i j}\right)$ for $(i, j) \in\{1,2,3\}^{2}, \delta_{d}=2\left(c_{d 11}-c_{d 12}\right)$ and $\gamma_{d}=$ $2\left(c_{d 11}-2 c_{d 12}+c_{d 23}\right)$, we have:

$$
q_{d}(x, y)=\delta_{d}\left(x^{2}+y^{2}\right)+\gamma_{d} x y-\delta_{d}(x+y)+c_{d 11} .
$$

In order to evaluate the coefficients $c_{d i j}$ we can decompose them using the contribution $c_{d i j}^{(u)}$ of each block $u$. For example when $i=j=1$, we have:

$$
c_{d 11}=\operatorname{tr}\left(C_{d 11}\right)=\operatorname{tr}\left(\sum_{u=1}^{b} T_{d u}^{\prime} Q_{k} T_{d u}\right)=\sum_{u=1}^{b} c_{d 11}^{(u)} \text { with } c_{d 11}^{(u)}=\operatorname{tr}\left(T_{d u}^{\prime} Q_{k} T_{d u}\right) .
$$

Simplifications of these forms give:

$$
c_{d 11}^{(u)}=k-\frac{n_{u}}{k}, c_{d 12}^{(u)}=m_{u}-\frac{n_{u}}{k}, c_{d 23}^{(u)}=p_{u}-\frac{n_{u}}{k},
$$

with:

$$
n_{u}=\sum_{i=1}^{t} n_{u i}^{2}, m_{u}=\sum_{i=1}^{t} m_{u i}, p_{u}=\sum_{i=1}^{t} p_{u i}
$$

denoting by $n_{u i}$ the number of plots where block $u$ receives treatment $i$, by $m_{u i}$ the number of times treatment $i$ is on the left side of itself for block $u$ and by $p_{u i}$ the number of plots having treatment $i$ on the left side and the right side for block $u$.

Denoting also $\delta_{d}^{(u)}=2\left(c_{d 11}^{(u)}-c_{d 12}^{(u)}\right)$ and $\gamma_{d}^{(u)}=2\left(c_{d 11}^{(u)}-2 c_{d 12}^{(u)}+c_{d 23}^{(u)}\right)$ we obtain finally the following decomposition over the blocks:

$$
q_{d}(x, y)=\sum_{u=1}^{b} h_{d}^{(u)}(x, y)
$$

where:

$$
h_{d}^{(u)}(x, y)=\delta_{d}^{(u)}\left(x^{2}+y^{2}\right)+\gamma_{d}^{(u)} x y-\delta_{d}^{(u)}(x+y)+c_{d 11}^{(u)}
$$

Two sequences of treatments in two blocks $u_{1}$ and $u_{2}$ are said to be equivalent if $\left(n_{u_{1}}, m_{u_{1}}, p_{u_{1}}\right)=\left(n_{u_{2}}, m_{u_{2}}, p_{u_{2}}\right)$, which is the case if one sequence is obtained from the other one by relabelling the treatments. By circularity of the design, it is also the case if one sequence may be obtained from the other one by a circular permutation of the plots. Therefore, for given $k$ and 
$t$, we can divide the set of all possible treatment sequences into $\mathcal{L}$ equivalence classes of treatments. Since $n_{u}, m_{u}$ and $p_{u}$ are the same for any $u$ in a given equivalence class, say $\ell$, we change the notation and write $n_{\ell}, m_{\ell}$ and $p_{\ell}$ instead. For a design $d$ we denote by $\pi_{d \ell}$ the proportion of blocks assigned to the class $\ell$. By abuse of notation $\ell$ refers to an equivalence class as well as its index, i.e. we can write $1 \leq \ell \leq \mathcal{L}$ and put:

$$
q_{d}(x, y)=b \sum_{\ell=1}^{\mathcal{L}} \pi_{d \ell} h_{\ell}(x, y)
$$

where:

$$
\begin{aligned}
h_{\ell}(x, y)= & 2\left(k-m_{\ell}\right)\left(x^{2}+y^{2}\right)+2\left(k-2 m_{\ell}+p_{\ell}\right) x y- \\
& 2\left(k-m_{\ell}\right)(x+y)+\left(k-\frac{n_{\ell}}{k}\right) .
\end{aligned}
$$

When $d$ is an exact design the proportions $\pi_{d \ell}$ are necessarily multiple of $1 / b$. If we remove this restriction we obtain an approximate design (sometimes called continuous block design).

\section{Optimal designs}

Consider the set $\Omega_{t, b, k}^{c}$ of all circular designs with $t$ treatments, $b$ blocks and $k$ plots. Our goal is now to obtain universally optimal designs. We know from Kiefer (1975) that a design $d^{*}$ for which the information matrix $C_{d^{*}}[\phi]$ is completely symmetric and that maximizes the trace of $C_{d}[\phi]$ over all the designs $d$ in $\Omega_{t, b, k}^{c}$ is universally optimal for the estimation of $\phi$. Thus, in order to obtain an approximate optimal design $d^{*}$ we have to find values $x^{*}$ and $y^{*}$ and proportions $\pi^{*}=\left(\pi_{d^{*} 1}, \ldots, \pi_{d^{*} \mathcal{L}}\right)$ such that $C_{d^{*}}[\phi]$ is completely symmetric and:

$$
q_{d^{*}}\left(x^{*}, y^{*}\right)=\max _{\pi} \min _{(x, y)} q_{d}(x, y)
$$

The following result (see Druilhet and Tinsson, 2009) shows that optimal designs may be seek among symmetric designs.

Proposition 1. For any circular design d, the information matrix for total effects $\phi=K^{\prime} \theta$ satisfies:

$$
\operatorname{tr} C_{d}[\phi] \leq \min _{(x, y)} q_{d}(x, y)
$$


and equality holds if the blocks $C_{d i j}$ of $C_{d}[\theta]$ are completely symmetric. In that case $C_{d}[\phi]$ is also a completely symmetric matrix.

The minimization of $q_{d}(x, y)$ may be reduce to the minimization of a one variable quadratic function using the following proposition:

Proposition 2. The value $q_{d}\left(x^{*}, y^{*}\right)$ is a minimum of the function $q_{d}(x, y)=$ $\operatorname{tr}\left(L^{* \prime} C_{d}[\theta] L^{*}\right)$ if and only if the two partial derivatives are equal to zero. Then we necessarily have:

$$
x^{*}=y^{*} .
$$

Proof. The first part of the proposition is due to the convexity of the function (see Lemma 3 in Druilhet and Tinsson, 2009). Using the form:

$$
q_{d}(x, y)=\delta_{d}\left(x^{2}+y^{2}\right)+\gamma_{d} x y-\delta_{d}(x+y)+c_{d 11}
$$

we obtain:

$$
\partial q_{d}(x, y) / \partial x=2 \delta_{d} x+\gamma_{d} y-\delta_{d} \text { and } \partial q_{d}(x, y) / \partial y=2 \delta_{d} y+\gamma_{d} x-\delta_{d}
$$

so the values $x^{*}$ and $y^{*}$ such that these two derivatives are zero are given by:

$$
x^{*}=y^{*}=\delta_{d} /\left(2 \delta_{d}+\gamma_{d}\right)
$$

Therefore, (3) can be simplified to:

$$
q_{d}(x)=b \sum_{\ell=1}^{\mathcal{L}} \pi_{d \ell} h_{\ell}(x)
$$

where:

$$
h_{\ell}(x)=2\left(3 k-4 m_{\ell}+p_{\ell}\right) x^{2}-4\left(k-m_{\ell}\right) x+\left(k-\frac{n_{\ell}}{k}\right) .
$$

The following proposition is an adaptation of Proposition 4 of Kunert and Martin (2000).

Proposition 3. Consider a symmetric design $d^{*} \in \Omega_{t, b, k}^{c}$ and a point $x^{*}$ such that the first derivative of $q_{d^{*}}$ is equal to zero. If we have:

$$
\forall \ell=1, \ldots, \mathcal{L}, b h_{\ell}\left(x^{*}\right) \leq q_{d^{*}}\left(x^{*}\right)
$$

then $d^{*}$ is universally optimal over $\Omega_{t, b, k}^{c}$. 
Proof. The design $d^{*}$ is symmetric so, from Proposition 1, the information matrix $C_{d^{*}}[\phi]$ is completely symmetric. Denote $q_{d}^{*}$ the minimum of the function $q_{d}$. For every design $d \in \Omega_{t, b, k}^{c}$ it is clear that $q_{d}^{*} \leq q_{d}\left(x^{*}\right)$ and:

$$
q_{d}\left(x^{*}\right)=b \sum_{\ell=1}^{\mathcal{L}} \pi_{d \ell} h_{\ell}\left(x^{*}\right) \leq b \sum_{\ell=1}^{\mathcal{L}} \pi_{d \ell} \frac{q_{d^{*}}\left(x^{*}\right)}{b} .
$$

We know from Proposition 2 that $q_{d^{*}}\left(x^{*}\right)=q_{d^{*}}^{*}$ and so we have proved that $d^{*}$ maximizes the trace of the information matrix over $\Omega_{t, b, k}^{c}$.

Now, for a given values of $t$ and $k$, an optimal designs for this onedimensional problem may be obtained using one class or a mixture of two different classes (see Kushner, 1997) and one of the following methods:

Method 1: to prove that the optimal design $d^{*}$ is generated by one treatment sequence $\ell_{1}$ (i.e. $q_{d^{*}}(x)=b h_{\ell_{1}}(x)$ ):

1) find $x^{*}$ that minimize $h_{\ell_{1}}$ and then the minimum $q_{d^{*}}^{*}$ of $q_{d^{*}}$,

2) check that for $1 \leq \ell \leq \mathcal{L}, b h_{\ell}\left(x^{*}\right) \leq q_{d^{*}}^{*}$.

Method 2: to prove that the optimal design $d^{*}$ is generated by two treatment sequences $\ell_{1}$ and $\ell_{2}$ (i.e. $\left.q_{d^{*}}(x)=b\left(\pi_{d^{*} \ell_{1}} h_{\ell_{1}}(x)+\pi_{d^{*} \ell_{2}} h_{\ell_{2}}(x)\right)\right)$ :

1) find an admissible intersection point $x^{*}$ according to the definition of Kusher (1997), that is:

$$
h_{\ell_{1}}\left(x^{*}\right)=h_{\ell_{2}}\left(x^{*}\right) \text { and } \frac{d h_{\ell_{1}}}{d x}\left(x^{*}\right) \frac{d h_{\ell_{2}}}{d x}\left(x^{*}\right) \leq 0,
$$

2) find the optimal proportions $\pi_{d^{*} \ell_{1}}^{*}$ and $\pi_{d^{*} \ell_{2}}^{*}$ such that $\left(d q_{d^{*}} / d x\right)\left(x^{*}\right)=$ 0 . So, denoting $a_{i}=\left(d h_{\ell_{i}} / d x\right)\left(x^{*}\right)$ for $i=1,2$ we obtain:

$$
\pi_{d^{*} \ell_{1}}^{*}=a_{2}\left(a_{2}-a_{1}\right)^{-1} \text { and } \pi_{d^{*} \ell_{2}}^{*}=a_{1}\left(a_{1}-a_{2}\right)^{-1},
$$

3) find the minimum $q_{d^{*}}^{*}=q_{d^{*}}\left(x^{*}\right)$ of $q_{d^{*}}(x)$,

4) check that for $1 \leq \ell \leq \mathcal{L}, b h_{\ell}\left(x^{*}\right) \leq q_{d^{*}}^{*}$.

Remark: A sequence is called degenerate when $h_{\ell}(x)$ is the null function for every $x$, that is when $\left(n_{\ell}, m_{\ell}, p_{\ell}\right)=\left(k^{2}, k, k\right)$ or equivalently when the sequence contains only a single treatment. It is easy to see that such sequence cannot appear in optimal designs and therefore will not be considered. Note that, if $q_{d}\left(x^{*}\right)=0$, then design $d$ is called degenerate. In that case, total effects are not estimable. 


\section{Optimal and efficient designs}

\subsection{Optimal approximate designs}

We give optimal designs in the sense of approximate design theory for several values of $k$ and $t$.

1) The case $k=3$. An optimal design can be generated by one or two sequences in the following set of non-degenerate sequences (for $t \geq 3$ ):

\begin{tabular}{|c|c|c|c|c||c|}
\hline$\ell$ & Sequence & $n_{\ell}$ & $m_{\ell}$ & $p_{\ell}$ & $h_{\ell}(x)$ \\
\hline 1 & {$\left[\begin{array}{lll}1 & 2 & 3\end{array}\right]$} & 3 & 0 & 0 & $18 x^{2}-12 x+2$ \\
\hline 2 & {$\left[\begin{array}{lll}1 & 1 & 2\end{array}\right]$} & 5 & 1 & 1 & $12 x^{2}-8 x+4 / 3$ \\
\hline
\end{tabular}

The minimum of the functions $h_{\ell}$ (for $\ell=1,2$ ), obtained for $x^{*}=1 / 3$, is equal to zero. Then it is impossible in that case to estimate the total effects (the size of the sequences is too small).

2) The case $k=4$. An optimal design can be generated by one or two sequences in the following set of non-degenerate sequences (for $t \geq 4$ ):

\begin{tabular}{|c|c|c|c|c|c||c|}
\hline$\ell$ & Sequence & $n_{\ell}$ & $m_{\ell}$ & $p_{\ell}$ & $h_{\ell}(x)$ \\
\hline 1 & {$\left[\begin{array}{llll}1 & 2 & 3 & 4\end{array}\right]$} & 4 & 0 & 0 & $24 x^{2}-16 x+3$ \\
\hline 2 & {$\left[\begin{array}{llll}1 & 2 & 1 & 3\end{array}\right]$} & 6 & 0 & 2 & $28 x^{2}-16 x+2.5$ \\
\hline 3 & {$\left[\begin{array}{llll}1 & 2 & 1 & 2\end{array}\right]$} & 8 & 0 & 4 & $32 x^{2}-16 x+2$ \\
\hline 4 & {$\left[\begin{array}{llll}1 & 1 & 2 & 3\end{array}\right]$} & 6 & 1 & 0 & $16 x^{2}-12 x+2.5$ \\
\hline 5 & {$\left[\begin{array}{llll}1 & 1 & 2 & 2\end{array}\right]$} & 8 & 2 & 0 & $8 x^{2}-8 x+2$ \\
\hline 6 & {$\left[\begin{array}{llll}1 & 1 & 1 & 2\end{array}\right]$} & 10 & 2 & 2 & $12 x^{2}-8 x+1.5$ \\
\hline
\end{tabular}

The minimum of the function $h_{1}$ is obtained for $x^{*}=1 / 3$ and it is equal to $q_{d^{*}}^{*}=b h_{\ell_{1}}\left(x^{*}\right)=b / 3$. Then it is easy to check that:

$$
\forall \ell=1, \ldots, 6, b h_{\ell}(1 / 3) \leq q_{d^{*}}^{*}
$$

So an optimal design is generated by the the sequence [ $\left.\begin{array}{llll}1 & 2 & 3 & 4\end{array}\right]$.

3) The case $k=5$ is developed in details in the Appendix. When $k$ is large, it is a tedious task to obtain explicit results, so optimal designs are obtained numerically. For $k \geq 5$, the following table gives the optimal designs. They are always generated by two treatment sequences and the optimal proportions are given in the second column. 


\begin{tabular}{|c|c|c|}
\hline$k$ & Optimal sequences & Proportions \\
\hline \multirow[t]{2}{*}{5} & $123445]$ & 0.0451 \\
\hline & 11234 & 0.9549 \\
\hline \multirow[t]{2}{*}{6} & $123456]$ & 0.0490 \\
\hline & 112234 & 0.9510 \\
\hline \multirow[t]{2}{*}{7} & 1112323 & 0.0990 \\
\hline & 1112223 & 0.9010 \\
\hline \multirow[t]{2}{*}{8} & 12123434 & 0.1271 \\
\hline & 11122233 & 0.8729 \\
\hline \multirow[t]{2}{*}{9} & 121234345 & 0.0918 \\
\hline & 111222333 & 0.9082 \\
\hline \multirow[t]{2}{*}{10} & 1112223434 & 0.2319 \\
\hline & 1111222333 & 0.7681 \\
\hline \multirow[t]{2}{*}{11} & 11112223434 & 0.1966 \\
\hline & 11112222333 & 0.8034 \\
\hline \multirow[t]{2}{*}{12} & 121234345656 & 0.0736 \\
\hline & 111222333444 & 0.9264 \\
\hline
\end{tabular}

For $k=5$, an optimal design may also be generated by the sequences [11223] and [12345] with proportions 0.4775 and 0.5225 . For $k=6$, optimal sequences may also be [112233] and [112345] with proportions 0.4510 and 0.5490 or [112233] and [123456] with proportions 0.6340 and 0.3660 or [112234] and [112345] with proportions 0.9019 and 0.0981. For $k=11$, optimal sequences may also be [11112222333] and [11123234545] with proportions 0.9017 and 0.0983

When the number $t$ of possible treatments is lower than the maximum number of treatments that appear in optimal sequences found above, we may use the same procedure described in Section 5 to obtain optimal designs but we consider only sequences having at most $t$ different treatments. For example, when $t=3$ and $k=8$, the optimal approximate design is generated by [11122233] and [11123232] with proportions 0.8571 and 0.1429 . 


\subsection{Efficient designs}

For a design $d$, we consider the classical $\Phi_{p}$ criteria (see e.g. Shah and Sinha, 1989) defined by:

$$
\Phi_{p}\left(C_{d}[\phi]\right)=\left(\frac{1}{t-1} \sum_{i=1}^{t-1} \lambda_{i}^{-p}\left(C_{d}[\phi]\right)\right)^{\frac{1}{p}},
$$

where $\lambda_{i}\left(C_{d}[\phi]\right)$ is the $i$ th greater eigenvalue of $C_{d}[\phi]$. For $p=-1, \Phi_{-1}\left(C_{d}[\phi]\right)=$ $\left[\operatorname{tr}\left(C_{d}[\phi]\right) /(t-1)\right]^{-1}$. For $p=0, p=1$ and $p=+\infty$, the $\Phi_{p}$ criteria are equivalent respectively to the well known $D$-, $A$ - and $E$ - criteria. The efficiency factor associated to a design $d$ and a criterion $\Phi_{p}$ is defined by

$$
\operatorname{eff}_{p}(d)=\frac{\Phi_{p}\left(C_{d^{*}}[\phi]\right)}{\Phi_{p}\left(C_{d}[\phi]\right)}
$$

where $d^{*}$ is an optimal approximate design having the same size as $d$. When $d$ is also symmetric in the sense defined in section $3.2, \Phi_{p}\left(C_{d}[\phi]\right)$ and eff $(d)$ do not depend on $p$ (see e.g. Druilhet, 2004) and therefore

$$
\text { eff }(d)=\frac{\operatorname{tr}\left(C_{d}[\phi]\right)}{\operatorname{tr}\left(C_{d^{*}}[\phi]\right)}=\frac{\operatorname{tr}\left(C_{d}[\phi]\right)}{q_{d^{*}}\left(x^{*}\right)} .
$$

The following table gives efficiency factors of CNBD2s in $\Omega_{t, b, k}^{c}$ for several values of $k$. Since a CNBD2 is a binary design, $t \geq k$. These designs are symmetric designs with all the treatment sequences in the same equivalence class $\ell=[1,2, \ldots, k]$. The trace of their information matrices are equal to $b(k-3) / 3$ (see Bailey and Druilhet, 2004).

\begin{tabular}{|c|c|c|c|c|c|c|c|c|c|}
\hline$k$ & 4 & 5 & 6 & 7 & 8 & 9 & 10 & 11 & 12 \\
\hline $\operatorname{Eff}\left(d^{*}\right)$ & 1 & 0.965 & 0.882 & 0.774 & 0.712 & 0.653 & 0.635 & 0.616 & 0.592 \\
\hline
\end{tabular}

Note that the lower bounds of efficiency factors obtained by Bailey and Druilhet (2004, Table 3) were quite lower than these obtained here since they used a cruder bound. We can see that CNBD2 are not very efficient when $k$ is large. In order to construct designs with reasonable number of blocks, we seek the most efficient symmetric design in $d \in \Omega_{t, b, k}^{c}$ generated by a single sequence, i.e. whose sequences belong to a single equivalence class, say $\ell$. When the number $t$ of treatments is greater or equal to the maximum number of treatments that appear in optimal sequences, then the efficiency factor of such a design is: 


$$
\operatorname{Eff}(d)=\frac{b h_{\ell}^{*}}{q_{d^{*}}\left(x^{*}\right)} \text { where } h_{\ell}^{*}=\min _{x} h_{\ell}(x)
$$

For several values of $k$, the following table gives the equivalence classes that maximize the efficiency factors of the corresponding designs.

\begin{tabular}{|c|c|c|}
\hline$k$ & Single sequence & Efficiency \\
\hline 5 & $\left.\begin{array}{lllll}1 & 1 & 2 & 3 & 4\end{array}\right]$ & 0.9999 \\
\hline 6 & 112234 & 0.9994 \\
\hline 7 & $\left.\begin{array}{lllllll}1 & 1 & 1 & 2 & 2 & 2 & 3\end{array}\right]$ & 0.9956 \\
\hline 8 & 111222234 & 0.9828 \\
\hline 9 & $\left.\begin{array}{llllllllll}1 & 1 & 1 & 2 & 2 & 2 & 3 & 3 & 3\end{array}\right]$ & 0.9797 \\
\hline 10 & 11122223334 & 0.9916 \\
\hline 11 & $\left.\begin{array}{lllllllllll}1 & 1 & 1 & 1 & 2 & 2 & 2 & 2 & 3 & 3 & 3\end{array}\right]$ & 0.9862 \\
\hline 12 & 111222333444 & 0.9874 \\
\hline
\end{tabular}

From a given equivalence class, a symmetric design may be obtained by considering all the treatment permutations. It can be obtained with $t$ ! / $(t-v)$ ! blocks, where $v$ is the minimum number of different treatments necessary to construct an optimal design.

When $t<v$, the optimal designs are obtained by procedure described in Section 5 but only with the sequences having at most $t$ treatments. Then the efficiency factors are obtained from optimal approximate designs that include this restriction as seen in Section 6.1, last paragraph. For example, for $k=5$, the optimal approximate design needs $v=5$ treatments since it contains the sequence $\left[\begin{array}{lllll}1 & 2 & 3 & 4 & 5\end{array}\right]$. If we have only 4 treatments, then we find that the optimal approximate design is generated by only one optimal sequence which is $\left[\begin{array}{lllll}1 & 1 & 2 & 3 & 4\end{array}\right]$. With $b=4$ ! blocks, the universally optimal design is therefore the exact design generated by this sequence:

$$
\left[\begin{array}{llllllllllllllllllllllll}
1 & 1 & 1 & 1 & 1 & 1 & 2 & 2 & 2 & 2 & 2 & 2 & 3 & 3 & 3 & 3 & 3 & 3 & 4 & 4 & 4 & 4 & 4 & 4 \\
1 & 1 & 1 & 1 & 1 & 1 & 2 & 2 & 2 & 2 & 2 & 2 & 3 & 3 & 3 & 3 & 3 & 3 & 4 & 4 & 4 & 4 & 4 & 4 \\
2 & 2 & 3 & 3 & 4 & 4 & 1 & 1 & 3 & 3 & 4 & 4 & 1 & 1 & 2 & 2 & 4 & 4 & 1 & 1 & 2 & 2 & 3 & 3 \\
3 & 4 & 2 & 4 & 2 & 3 & 3 & 4 & 1 & 4 & 1 & 3 & 2 & 4 & 1 & 4 & 1 & 2 & 2 & 3 & 1 & 3 & 1 & 2 \\
4 & 3 & 4 & 2 & 3 & 2 & 4 & 3 & 4 & 1 & 3 & 1 & 4 & 2 & 4 & 1 & 2 & 1 & 3 & 2 & 3 & 1 & 2 & 1
\end{array}\right]
$$

where the rows correspond to the plots and the columns to the blocks. The guard plots have been omitted. 


\section{Appendix}

We develop here the case $k=5$. We have for each sequence:

$$
h_{l}(x)=2\left(15-4 m_{l}+p_{l}\right) x^{2}-4\left(5-m_{l}\right) x+\left(5-\frac{n_{l}}{5}\right) .
$$

An optimal design can be generated by one or two sequences in the following set of non-degenerate sequences (for $t \geq 5$ ):

\begin{tabular}{|c|c|c|c|c|c||c|}
\hline$l$ & Sequence & $n_{l}$ & $m_{l}$ & $p_{l}$ & $h_{l}(x)$ \\
\hline 01 & {$\left[\begin{array}{lllll}1 & 2 & 3 & 4 & 5\end{array}\right]$} & 5 & 0 & 0 & $30 x^{2}-20 x+4$ \\
\hline 02 & {$\left[\begin{array}{lllll}1 & 2 & 1 & 3 & 4\end{array}\right]$} & 7 & 0 & 1 & $32 x^{2}-20 x+3.6$ \\
\hline 03 & {$\left[\begin{array}{lllll}1 & 1 & 2 & 3 & 4\end{array}\right]$} & 7 & 1 & 0 & $22 x^{2}-16 x+3.6$ \\
\hline 04 & {$\left[\begin{array}{lllll}1 & 2 & 1 & 2 & 3\end{array}\right]$} & 9 & 0 & 2 & $34 x^{2}-20 x+3.2$ \\
\hline 05 & {$\left[\begin{array}{lllll}1 & 1 & 2 & 3 & 2\end{array}\right]$} & 9 & 1 & 1 & $22 x^{2}-16 x+3.2$ \\
\hline 06 & {$\left[\begin{array}{lllll}1 & 1 & 2 & 2 & 3\end{array}\right]$} & 9 & 2 & 0 & $14 x^{2}-12 x+3.2$ \\
\hline 07 & {$\left[\begin{array}{lllll}1 & 1 & 2 & 1 & 3\end{array}\right]$} & 11 & 1 & 1 & $24 x^{2}-16 x+2.8$ \\
\hline 08 & {$\left[\begin{array}{lllll}1 & 1 & 1 & 2 & 3\end{array}\right]$} & 11 & 2 & 1 & $16 x^{2}-12 x+2.8$ \\
\hline 09 & {$\left[\begin{array}{lllll}1 & 1 & 2 & 1 & 2\end{array}\right]$} & 13 & 1 & 3 & $28 x^{2}-16 x+2.4$ \\
\hline 10 & {$\left[\begin{array}{lllll}1 & 1 & 1 & 2 & 2\end{array}\right]$} & 13 & 3 & 1 & $8 x^{2}-8 x+2.4$ \\
\hline 11 & {$\left[\begin{array}{lllll}1 & 1 & 1 & 1 & 2\end{array}\right]$} & 17 & 3 & 3 & $12 x^{2}-8 x+1.6$ \\
\hline
\end{tabular}

From Figure 1 an admissible intersection point $x^{*}$ seems to be associated to the two sequences [12345] and [11234] (in bold on the figure).

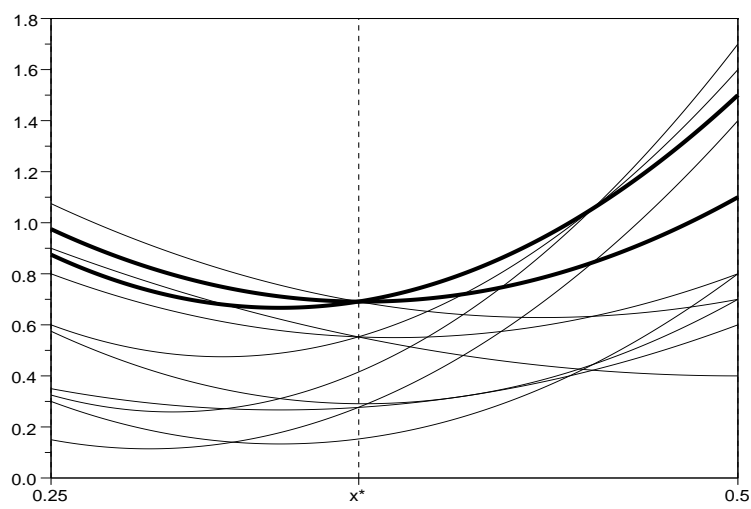

Fig. 1. Graphical representation of $h_{l}$ for $l=1, \ldots, 11$.

Functions $h_{1}$ and $h_{3}$ are in bold. 
Such point is explicitely given by:

$$
h_{1}\left(x^{*}\right)=h_{3}\left(x^{*}\right) \Leftrightarrow x^{*}=\frac{1+\sqrt{0.2}}{4} .
$$

(the inequality $\left(d h_{1} / d x\right)\left(x^{*}\right)\left(d h_{2} / d x\right)\left(x^{*}\right) \leq 0$ hold). Then we obtain the proportions:

$$
\pi_{d^{*} 1}^{*}=\frac{\frac{d h_{3}}{d x}\left(x^{*}\right)}{\frac{d h_{3}}{d x}\left(x^{*}\right)-\frac{d h_{1}}{d x}\left(x^{*}\right)}=\frac{5-11 \sqrt{0.2}}{4 \sqrt{0.2}} \text { and } \pi_{d^{*} 3}^{*}=\frac{-5+15 \sqrt{0.2}}{4 \sqrt{0.2}} .
$$

Finally we easily check that:

$$
\forall l=1, \ldots, 11, b h_{l}\left(x^{*}\right) \leq q_{d^{*}}^{*}
$$

with:

$$
q_{d^{*}}^{*}=q_{d^{*}}\left(x^{*}\right)=b\left(\pi_{d^{*}}^{*} h_{1}\left(x^{*}\right)+\pi_{d^{*} 3}^{*} h_{3}\left(x^{*}\right)\right)=\frac{5 b}{4}(1-\sqrt{0.2}) .
$$

So these two sequences with proportions $\pi_{d^{*} 1}^{*}$ and $\pi_{d^{*} 3}^{*}$ generate an optimal design. Note that an optimal design may also be generated by the sequences [12345] and [11223] with proportions $\frac{1}{8}(5 \sqrt{5}-7)$ and $\frac{5}{8}(3-\sqrt{5})$ but not by a combination of [11234] and [11223] since the derivatives of $h_{3}(x)$ and $h_{6}(x)$ have the same sign at $x=x^{*}$.

\section{References}

[1] Bailey, R. A., Druilhet, P., 2004. Optimality of neighbor-balanced designs for total effects. Ann. Statist. 32 (4), 1650-1661.

[2] Besag, J., Kempton, R. A., 1986. Statistical analysis of field experiments using neighbouring plots. Biometrics 42, 231-251.

[3] Druilhet, P., 1999. Optimality of neighbour balanced designs. J. Statist. Plann. Inference 81 (1), 142-152.

[4] Druilhet, P., 2004. Conditions for optimality in experimental designs. Linear Algebra Appl. 388, 147-157. 
[5] Druilhet, P., Tinsson, W., 2009. Optimal cross-over designs for a model with partial interaction. Biometrika 96, 977-690.

[6] Filipiak, K., Markiewicz, A., 2005. Optimality and efficiency of circular neighbor balanced designs for correlated observations. Metrika 61 (1), $17-27$.

[7] Gaffke, N., 1987. Further characterizations of design optimality and admissibility for partial parameter estimation in linear regression. Ann. Statist. 15 (3), 942-957.

[8] Kempton, R. A., 1985. Spatial methods in fields experiments. Biometric Bulletin 2, 4-5.

[9] Kempton, R. A., 1997. Interference between plots. In: Kempton, R. A., Fox, P. N. (Eds.), Statistical Methods for Plant Variety Evaluation. Chapman and Hall, London, pp. 101-116.

[10] Kiefer, J., 1975. Construction and optimality of generalized Youden designs. In: A survey of statistical design and linear models (Proc. Internat. Sympos., Colorado State Univ., Ft. Collins, Colo., 1973). NorthHolland, Amsterdam, pp. 333-353.

[11] Kunert, J., 1983. Optimal design and refinement of the linear model with applications to repeated measurements designs. Ann. Statist. 11 (1), $247-257$.

[12] Kunert, J., 2001. Interference designs with circular structure. In: Kunert, J., Trenkler, G. (Eds.), Mathematical Statistics with Applications in Biometry. Eul Verlag, pp. 355-368.

[13] Kunert, J., Martin, R. J., 2000. On the determination of optimal designs for an interference model. Ann. Statist. 28 (6), 1728-1742.

[14] Kushner, H. B., 1997. Optimal repeated measurements designs: the linear optimality equations. Ann. Statist. 25 (6), 2328-2344.

[15] McGilchrist, C. A., Trenbath, B. R., 1971. A revised analysis of competition experiments. Biometrics 27, 659-671.

[16] Shah, K. R., Shinha, B. K., 1989. Theory of optimal designs. Lecture Notes in Statistics, Springer, New York. 
[17] Pukelsheim, F., 1993. Optimal design of experiments. Wiley Series in Probability and Mathematical Statistics: Probability and Mathematical Statistics. John Wiley \& Sons Inc., New York. 